included in the study. Following the clinical assessment of the patient, serum cancer antigen 125 levels and an ultrasound pelvis/abdomen were done along with the other investigations needed for management. The findings were then classified as benign or malignant as per the various RMI classification systems using a cut-off of 200 for RMI-1, 2, 3 and that of 450 for RMI-4. The results were compared with the histopathology report obtained postoperatively.

Results: RMI-1 has a sensitivity of $70 \%$, specificity of $85.42 \%$ and AUC was 0.85, and accuracy of 82.76. RMI-2 had a sensitivity of $80 \%$, specificity of $82.64 \%$ with AUC of 0.856 , and accuracy of 82.18. RMI-3 had findings similar to that of RMI-1 despite their University System of Georgia scores being different. But a had an AUC of 0.84. RMI-4 had a sensitivity of $90 \%$, Specificity of $77.08 \%$, and highest AUC of 0.87 , and lowest accuracy of 79.31 .

Conclusion: All the RMI scores showed comparable sensitivity and specificity. However, RMI-2 had slightly better sensitivity and specificity as compared to the rest. The addition of tumor size to the RMI-4 classification increases the sensitivity at the cost of specificity. In low-resource settings or developing countries, RMI is the most commonly used system for classifying adnexal masses as benign or malignant.

Oral (OMi9)

Miscellaneous

https://doi.org/10.3802/jgo.2021.32.S1.0Mi9

\section{Peripheral lymphocytes reflect exhausted immune phenotypes of tumor-infiltrating lymphocytes in endometrial cancer patients}

Yup Kim, Miran Lee, JooHyang Lee, Yong-Jae Lee, Sung Hoon Kim, Sang Wun Kim, Jung-Yun Lee, Junsik Park

Yonsei University College of Medicine, Seoul, Korea (byjspark@yuhs.ac)
Objective: The aims of this study was to characterize exhausted immune phenotypes of tumor-infiltrating lymphocytes (TILs) in endometrial cancer patients and investigate their clinical relevance. Methods: We isolated peripheral blood mononuclear cells (PBMCs) and TILs from 36 patients with newly diagnosed endometrial cancer and examined their composition, differentiation, and exhaustion status (expression of immune checkpoint receptors and T-cell transcription factors) using multicolor flow cytometry. Results: The percentage of CD3 T cells in $\mathrm{CD} 45^{+}$hematopoietic cells were increased in TILs. Unlike CD4 T cells, the proportion of CD8 T cells and regulatory T cells (Treg) were significantly increased in TILs. Considering the CD8/Treg ratio, the increase in Tregs was more pronounced. When analyzing differentiation subsets using the markers CCR7 and CD45RA, effector memory $\mathrm{T}$ cells (CCR7 CD45RA ${ }^{-}$) were enriched in TILs. Immune checkpoint receptors (PD-1, CTLA-4, TIM-3, and TIGIT) were expressed more on CD8 T cells and Tregs in TILs than in PBMC. Intriguingly, although TIGIT $^{+}$cells were increased in CD8 TILs, the percentage of $\mathrm{CD} 226^{+} \mathrm{TIGIT}^{+}$cells among CD8 T cells were significantly decreased in TILs. Comparing proliferation marker (Ki-67), stemlike property (T cell factor-1; TCF-1), and exhaustion marker (TOX) between peripheral CD8 T cells and CD8 TILs, CD8 TILs showed higher expression of Ki-67 and TOX, and lower expression of TCF-1. In the case of Tregs, tumor-infiltrating Tregs showed higher expression of activation marker $\mathrm{CD} 39$, antigen-reactive marker 4-1BB, and proliferation marker Ki-67 than peripheral Tregs. Next, we analyzed correlation of immune profiles between peripheral $\mathrm{T}$ cells and TILs. The expression of PD-1, CTLA-4, 4-1BB, and CD39 showed significant correlation between peripheral and tumorinfiltrating Tregs. In the case of CD8 T cells, only PD-1 expression showed a significant correlation between PBMCs and TILs. Conclusion: In endometrial cancer, tumor-infiltrating lymphocytes were exhausted and had suppressive properties. CD4 and CD8 TILs showed exhausted immune-phenotypes and tumor-infiltrating Tregs. Furthermore, peripheral lymphocytes reflect these immunephenotypes of TILs in endometrial cancer patients. 